University of Nebraska - Lincoln

DigitalCommons@University of Nebraska - Lincoln

Papers in Reaction Kinetics

Chemical and Biomolecular Engineering

Research and Publications

5-1-1998

\title{
Analysis of combustion-driven acoustics
}

L Boshoff-Mostert

Department of Chemical Engineering, University of Nebraska-Lincoln

Hendrik J. Viljoen

University of Nebraska-Lincoln, hviljoen1@unl.edu

Follow this and additional works at: https://digitalcommons.unl.edu/chemengreaction

Part of the Chemical Engineering Commons

Boshoff-Mostert, L and Viljoen, Hendrik J., "Analysis of combustion-driven acoustics" (1998). Papers in Reaction Kinetics. 10.

https://digitalcommons.unl.edu/chemengreaction/10

This Article is brought to you for free and open access by the Chemical and Biomolecular Engineering Research and Publications at DigitalCommons@University of Nebraska - Lincoln. It has been accepted for inclusion in Papers in Reaction Kinetics by an authorized administrator of DigitalCommons@University of Nebraska - Lincoln. 


\title{
Analysis of combustion-driven acoustics
}

\author{
L. Boshoff-Mostert and H. J. Viljoen* \\ Department of Chemical Engineering, University of Nebraska-Lincoln, Lincoln, NE 68588- \\ 0126, U.S.A.
}

\begin{abstract}
Combustion-driven acoustic oscillations are investigated by performing a onedimensional stability analysis of a burner-stabilized premixed flame. In contrast to other investigators, no initial acoustic wave is assumed in the analysis; the downstream acoustic field results from flame instability. Two models are considered: the thermodiffusive model (uncoupled model) and the fully coupled thermodiffusive-hydrodynamic model. The fully coupled problem exhibits instability at a much lower critical Lewis number than the uncoupled problem.
\end{abstract}

\section{INTRODUCTION}

Flames stabilized on a flame holder or inside a combustion chamber may become unstable towards infinitesimally small perturbations and pressure oscillations will build up and an intense sound may be emitted. Combustion-driven acoustic oscillations are important in a number of combustor applications, including jet and rocket engines and pulse combustors. Because of the destructive consequences that can $r$ jult from acoustic instability, the phenomenon has been and continues to be extensively studied ( $\mathrm{Si}$ powicz et al., 1971; Lawn, 1982). A low-frequency instability can lead to intense pressure perturbations in afterburners of aerojet engines (Macquisten and

Dowling, 1995). In propulsion systems large amplitude oscillations can result in unacceptable structural vibrations and even failure (Margolis, 1993).

A number of papers addressed the acoustic problem from a different point of view. A pressure field, modulated externally up stream of the flame is imposed on the system, and the stability of the field downstream of the flame is analyzed. Toong et al. (1975) investigated the acoustic-kinetic interaction between an acoustic wave and chemical reaction. An initially right-traveling plane acoustic wave in a gaseous medium is subject to a simple one-step exothermic Arrhenius-type reaction. They found that acoustic-kinetic coupling can, depending on the system conditions, lead to amplification or attenuation of the acoustic field. In a similar study of acoustic-kinetic interaction. Garris et al. (1975) found that reac-

*Corresponding author. Tel.: (402) 472-9318. fax: (402) 173-6989. tant consumption may be retarded or accelerated, depending upon the initial dimensionless acoustic frequency of the disturbance. Gilbert er al. (1973) studied the acoustic-kinetic interaction of a standing acoustic wave in a gaseous medium. They found for the decomposition of cyclopropane at $953 \mathrm{~K}$, acoustic waves with frequency of $500 \mathrm{~Hz}$.

Clavin et al. (1990) analyzed the stability limits of vibratory instabilities of a planar fiame propagating in a tube. The origin of the instability comes from time-dependent modifications of the flame structure which are induced by acoustic waves. McIntosh (1987) studied the interaction of a one-dimensional flame with long wavelength acoustic waves when the flame is anchored to a perforated plate within a tube.

This analysis differs from previous analysis because the downstream acoustic field is the result of flame instability. No acoustic wave is introduced initially. When a planar flame becomes unstable via a Hopf bifurcation, the flame oscillates around the steadystate position. An oscillatory flame can lead to pressure oscillations both upstream and downstream of the flame sheet. The frequency of the acoustic pressure field is determined by the frequency of the flame instability. Experimental proof of oscillations in the audible range is well documented (Sugimoto and Matsui, 1982; Afanas'ev et al., 1995).

Two descriptions of the combustion problem are analyzed in the linear stability analysis. The first description follows the thermodiffusive model where an isobaric assumption allows the temperature and concentration solutions to be found independent of the density, pressure and fluid velocity. This is referred to as the uncoupled case. The second description includes a time derivative of pressure in the energy balance which couples the momentum and energy 
equations. Due to the coupling the temperature. concentration, pressure, density and fluid velocity equations need to be solved simultanously. The important effect of the coupled model is that flame instability is found at lower Lewis numbers.

In this study the frequency of the pressure perturbations is related to the inlet fuel fraction for both the coupled and uncoupled case at two different burner temperatures. The downstream pressure perturbation is shown to form a standing wave pattern on the acoustic length scale.

\section{MODEL}

A premixed flame, stabilized on a cooled burner, is considered. This analysis considers only the axial spatial variable. To facilitate the analysis, we assume that the combustion chamber is in thermal equilibrium with the gas phase at all times. Hence, there is no radial variation in the gas-phase temperature field. A mixture of air and fuel is fed to the burner and the flame is stabilized at a small distance from the burner surface. This stand-off distance is determined by physicochemical properties of the system.

At steady state the stand-off distance remains constant, but for certain parameter values, the steadystate solution becomes unstable via a Hopf bifurcation. Beyond the critical point, the stand-off distance becomes a sinusoidal function in time which causes the flame sheet to oscillate around the steady state position. If it is recognized that the flame sheet drives pressure perturbations downstream, it is acceptable to think of the flame sheet as a vibrating diaphragm. (Actually, the flame sheet behaves in the opposite manner as a diaphragm in the sense that the downstream pressure increases when a diaphragm moves downstream. whilst the pressure downstream of the flame sheet decreases when the flame sheet moves downstream, due to the increase in temperature.)

To distinguish between conditions at the burner and at the flame sheet, the subscripts $u$ and $\mathrm{b}$ are used, respectively. The subscript $a$ is used for adiabatic values and the subscript $\mathrm{c}$ is used to identify variables at the bifurcation point. In this analysis, we only consider subsonic flow (small Mach numbers). Since flame destabilization occurs at velocities below the adiabatic flame velocity, this is not a restrictive assumption.

The ideal gas law is used as the equation of state:

$$
P=\rho R_{m} T
$$

where $\mathrm{R}$, is taken as $\mathrm{R}, \times M_{\mathrm{air}}$. The continuity equation is

$$
\frac{\partial \rho}{\partial t}+\frac{\partial(\rho U)}{\partial x}=0
$$

and the momentum balance is given by

$$
\rho \frac{\partial U}{\partial t}+\rho U \frac{\partial U}{\partial x}+\frac{\partial P}{\partial x}=0 .
$$

The species and energy balances are

$$
\begin{gathered}
\frac{\partial C}{\partial t}+\frac{\partial(C U)}{\partial x}+k C \mathrm{e}^{-E / R_{.} r}=D \frac{\partial^{2} C}{\partial x^{2}} \\
\rho C_{r} \frac{\partial T}{\partial t}+\rho U C_{r} \frac{\partial T}{\partial x} \\
=k_{c} \frac{\partial^{2} T}{\partial x^{2}}+(-\Delta H) k C \mathrm{e}^{-E / R_{m} T}+\frac{\partial P}{\partial t}+u \frac{\partial P}{\partial x} .
\end{gathered}
$$

To keep the focus on the acoustic aspect, intricacies which arise from conditions of near stoichiometric mixtures will be neglected (Margolis, 1980) - it is assumed that the mixture is sufficiently nonstoichiometric that $\mathrm{C}$ describes the limiting species. Furthermore, a single step first-order reaction is used to describe the kinetics.

It is also assumed that the product of the diffusion coefficient and density, $\mathrm{D} \times \mathrm{p}$ is constant. In his analysis, Margolis used a density-weighted axial variable (useful to eliminate the continuity equation) and assumed that $\mathrm{D} \times \rho^{2}$ and $\mathrm{k}, \times p$ are constant $(k$, is the thermal conductivity). We did not choose to use a density-weighted axial variable, leaving us with a variable coefficient eigenvalue problem. We assume that the density in the region between the burner and the flame remains constant, and we use the value of the density in the flame in the analysis (Joulin and Clavin, 1979). This assumption removes the variable coefficients from the eigenvalue problem. This assumption is discussed in detail in the uncoupled problem section (Section 3.1).

Equations (1)-(5) are written in dimensionless form; pressure, density and temperature are scaled with respect to the adiabatic values, velocity is scaled with respect to the adiabatic flame velocity $U$, and concentration is scaled by the far upstream value $C_{\text {in }}$. Let $\mathrm{p}$, $\mathrm{m}$ and $\theta$ denote the dimensionless pressure, density and temperature and $u, \mathrm{Y}$ denote the dimensionless velocity and fuel mass fraction. The thermal diffusivity is denoted as $K$ and we assume that it remains constant. Time and position are scaled as $\tau=t U_{u}^{2} / \kappa$, and $z=x U_{u} / \kappa$, respectively. The feed rate of the gas mixture, $\rho_{\text {in }} U_{\text {in }}$ is constant and in terms of the scales $p$, and $U$, it is denoted as the dimensionless flux $x_{F}$. The non-dimensional equations are

$$
\begin{aligned}
& p=m \theta \\
& \frac{d t n}{\partial \tau}+\frac{\partial(m u)}{\partial z}=0 \\
& m \frac{\partial u}{\partial \tau}+m u \frac{\partial u}{\partial z}+x_{1} \frac{\partial p}{\partial z}=0 \\
& m \frac{\partial Y}{\partial \tau}+m u \frac{\partial Y}{\partial z}+m D a Y \mathrm{e}^{\left.\gamma\left(\mathbf{1}_{i} \theta_{t}\right)-1 / \theta\right]}=\frac{1}{L e} \frac{\partial^{2} Y}{\partial z^{2}} \\
& m \frac{\partial \theta}{\partial \tau}+m u \frac{\partial \theta}{\partial z}=\frac{\partial^{2} \theta}{\partial z^{2}} \\
& +\left(1-\theta_{u}\right) m D a Y \mathrm{e}^{\left[\because\left(1 \theta_{n}\right)-1 \theta\right]}+\frac{R}{C,}\left(\frac{\partial p}{\partial \tau}+\frac{\partial p}{\partial z}\right) .
\end{aligned}
$$


The downstream constitutive continuity and momentum equations are coupled to the energy balance in the eigenvalue problem due to the $\left(R / C_{p}\right)[(\partial p / \partial \tau)$ $+(\partial p / \partial z)]$ term. Since the flame velocity is significantly less than the speed of sound, effects due to the compressibility of the gas may be neglected (Sivashinsky, 1983). In both the coupled and uncoupled case the compressibility effects associated with pressure variations in the energy equation $(\partial p / \partial z)$ are neglected. Clavin et al. (1990) showed that the isobaric assumption is well justified for small Mach numbers. Also consider the steady-state momentum equation (Williams, 1985): if we assume $\mathrm{u}^{2} /(\mathrm{p} / \mathrm{m}) \ll 1$ (small Mach number) then the equation

$$
m u \frac{\mathrm{d} u}{\mathrm{~d} z}+x_{1} \frac{\mathrm{dp}}{\mathrm{dz}}=0
$$

reduces to

$$
\frac{\mathrm{d} \ln p}{\mathrm{~d} z} \ll \frac{\mathrm{d} \ln u}{\mathrm{~d} z} .
$$

We see that fractional changes in the pressure are negligibly small. We solve both the coupled case (shown above) and the uncoupled case where $\partial p / \partial \tau=0$.

The boundary conditions at the inlet (burner surface) are

$$
\begin{gathered}
\theta=\theta_{u} \\
\frac{\partial Y}{\partial z}=x_{F} L e[Y-1] .
\end{gathered}
$$

A Danckwert's-type condition is imposed for the reactant, but the temperature is equal to the burner face temperature. implying a non-adiabatic condition. For the temperature it is sufficient to require that the downstream temperature is bounded. The fuel is completely consumed by the flame, and Y is identically zero downstream. The origin of the coordinate system is defined as the steady-state flame position, $z=0$. Flames are associated with large activation energies. reflected by the parameter $\%$. In the asymptotic limit the flame structure is made up of two different length scales; an upstream (and downstream) non-reactive zone and a very thin reaction-diffusion region (flame sheet). Outside the flame sheet the reaction term can be neglected.

It can be shown (Margolis, 1980) by matched asymptotic analysis of the flame sheet that the reaction term can be replaced by a source term located at the flame position.

$$
m D a Y \mathrm{e}^{\left.\gamma\left(1 / / \sigma_{b}\right)-1 / 0\right]}=\delta_{f} \chi_{F} \mathrm{e}^{\left(2 / 2 \theta_{b}^{2}\right)\left(0-\theta_{b}\right)}
$$

where $\delta_{f}$ is the Kronecker delta function andf denotes the instantaneous flame sheet position. At the flame sheet, $z=f$, the following continuity and jump conditions hold:

$$
\begin{aligned}
& {[\theta]=0} \\
& {[p]=0} \\
& {[u]=0}
\end{aligned}
$$

\subsection{Steady-state solution}

The steady-state solutions (subscript o) upstream and downstream of the flame sheet are distinguished by the use of the superscripts - and + , respectively. The upstream solutions are

$$
\begin{gathered}
\theta_{o}^{-}=\frac{\mathrm{B},-\theta_{b} \mathrm{e}^{-x_{F} h}+\left(\theta_{b}-\theta_{u}\right) \mathrm{e}^{x_{F} z}}{1-\mathrm{e}^{-x_{F} h}} \\
Y_{o}^{-}=1-\mathrm{e}^{x_{f} L e z} \\
m^{-}=\frac{1-\mathrm{e}^{-x_{F} h}}{\mathrm{~B},-\theta_{b} \mathrm{e}^{-x_{f} h}+\left(\theta_{b}-\theta_{u}\right) \mathrm{e}^{x_{F} z}} \\
u_{o}^{-}=\frac{x_{F}\left[Q,-\theta_{h} \mathrm{e}^{-x_{F} h}+\left(\theta_{b}-\theta_{u}\right) \mathrm{e}^{x_{F} z}\right]}{1-\mathrm{e}^{-x_{F} h}} \\
p_{o}^{-}=1 .
\end{gathered}
$$

The downstream solutions are

$$
\begin{aligned}
\theta_{o}^{+} & =\theta_{b} \\
Y_{o}^{+} & =0 \\
m_{o}^{+} & =\frac{1}{\theta_{h}} \\
u_{o}^{+} & =x_{F} \theta_{b} \\
p_{0}^{+} & =1 .
\end{aligned}
$$

\section{LINEAR STABILITY ANALYSIS}

The problem is solved by perturbation series and the following variables are introduced:

$$
\begin{aligned}
\theta & =\theta_{o}+\varepsilon \theta_{1}+\cdots \\
Y & =Y_{o}+\varepsilon Y_{1}+\cdots \\
m & =m_{o}+\varepsilon m_{1}+\cdots \\
u & =u_{o}+\varepsilon u_{1}+\cdots \\
p & =p_{o}+\varepsilon p_{1}+\cdots \\
f & =\varepsilon f_{1}+\cdots,
\end{aligned}
$$

where $\varepsilon$ is a small parameter. The above perturbations are applied to eqs $(6)-(18)$. The leading order $[O(1)]$ perturbation problem yields the steady-state solution. The next order of terms $[O(\varepsilon)]$ lead to the eigenvalue problem with the generic form $s_{1}(z) \mathrm{e}^{\omega \tau}$. The instantaneous flame position is at

$$
z=f=\varepsilon f_{1}=f_{11} \mathrm{e}^{\omega r}
$$

The stand-off distance $\mathrm{h}$ can be determined by integrating the steady-state forms of eqs (9) and (10) across the flame sheet:

$$
\mathrm{e}^{x_{f} h}=\frac{1-\theta_{u}}{1-\bar{\theta}_{b}}
$$


In Fig. 1 the stand-of distance is plotted as a function of $\theta_{h}$ for different methane fractions (parameter values as listed in Table 1). For some of the values of $h$ there are two $\theta_{h}$ values. Lower inlet fuel concentrations have larger stand-of distances. The curves show an increase as the adiabatic value is approached and $\mathrm{h} \rightarrow \infty$ when $\theta \rightarrow 1$, indicating that the adiabatic value can only be reached when the flame is infinitely far away from the heat sink (burner face).

\subsection{Uncoupled problem}

The eigenvalue problem $[O(\varepsilon)]$ associated with the uncoupled equations $(6)-(10)$ is

$$
\begin{gathered}
m_{1} \theta_{o}+m_{o} \theta_{1}=p_{1} \\
\omega m_{1}+\frac{\mathrm{d}\left(m_{1} u_{o}+m_{o} u_{1}\right)}{\mathrm{d} z}=0 \\
m_{o}\left[\omega u_{1}+u_{o} \frac{\mathrm{d} u_{1}}{\mathrm{~d} z}\right]+\left(m_{1} u_{o}+m_{o} u_{1}\right) \frac{\mathrm{d} u_{o}}{\mathrm{~d} z} \\
\omega m_{o} Y_{1}+\left(m_{0} u_{1}+m_{1} u_{o}\right) \frac{\mathrm{d} Y_{o}}{\mathrm{~d} z}+x \frac{\mathrm{d} Y_{1}}{\mathrm{~d} z} \\
=\frac{1}{L e} \frac{\mathrm{d}^{2} Y_{1}}{\mathrm{~d} z^{2}} \\
m_{v} \omega \theta_{1}+x \frac{\mathrm{d} \theta_{1}}{\mathrm{~d} z}=\frac{\mathrm{d}^{2} \theta_{1}}{\mathrm{~d} z^{2}} .
\end{gathered}
$$$$
m_{o}\left[\omega u_{1}+u_{o} \frac{\mathrm{d} u_{1}}{\mathrm{~d} z}\right]+\left(m_{1} u_{o}+m_{0} u_{1}\right) \frac{\mathrm{d} u_{o}}{\mathrm{~d} z}+\alpha_{1} \frac{\mathrm{d} p_{1}}{\mathrm{~d} z}=0
$$

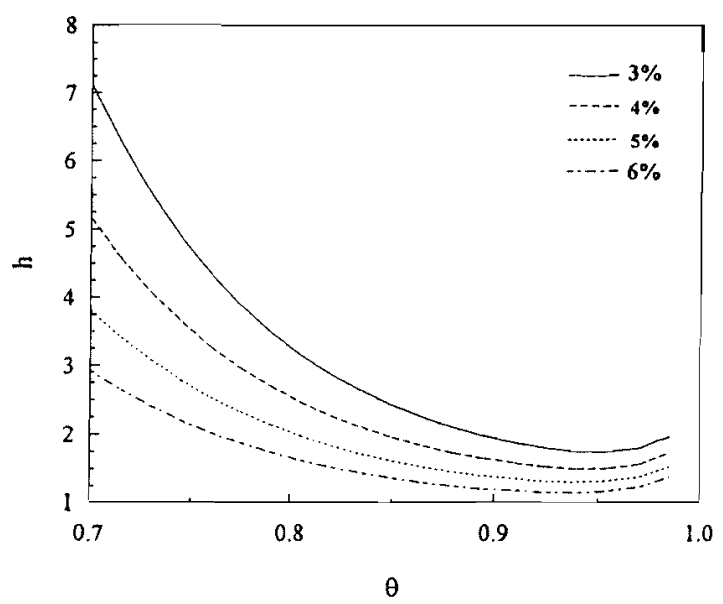

Fig. 1. Stand-of distance $(h)$.

Table 1. Properties for methane combustion

\begin{tabular}{ccc}
\hline Item & Units & Value \\
\hline$C p_{y}$ & $\mathrm{~J} / \mathrm{mol} \mathrm{K}$ & 40 \\
$E$ & $\mathrm{~J} / \mathrm{mol}$ & 130,000 \\
$\Delta H$ & $\mathrm{~J} / \mathrm{mol}$ & $8 \times 10^{5}$ \\
$k_{\mathrm{c}}$ & $\mathrm{W} / \mathrm{m} \mathrm{K}$ & 0.15 \\
$k_{v}$ & $1 / \mathrm{s}$ & $1.8 \times 10^{8}$ \\
$R_{y}$ & $\mathrm{~J} / \mathrm{mol} \mathrm{K}^{3}$ & 8.314 \\
$\rho_{f}$ & $\mathrm{~kg} / \mathrm{m}^{3}$ & $P M / R_{y} T$ \\
\hline
\end{tabular}

At the flame sheet the following contunuty and jump conditions hold:

$$
\begin{gathered}
{\left[\theta_{1}\right]=0} \\
{\left[Y_{1}\right]=0} \\
{\left[\frac{\mathrm{d} \theta_{1}}{\mathrm{~d} z}\right]+\left(1-\theta_{u}\right) x_{F} \frac{\gamma \theta_{1}^{+}}{2 \theta_{b}^{2}}=0} \\
{\left[\frac{\mathrm{d} Y_{1}}{\mathrm{~d} z}\right]=x_{F} L e \frac{\gamma \theta_{1}^{+}}{2 \theta_{b}^{2}}} \\
{\left[u_{1}\right]=0} \\
{\left[m_{1}\right]=0 .}
\end{gathered}
$$

It follows from the equation of state and continuity of $u_{1}$ and $m$, that $p$, is also continuous. At the inlet. $z=-h$, the boundary conditions become

$$
\begin{gathered}
\theta_{1}=0 \\
Y_{1}-\frac{1}{x_{F} L e} \frac{\mathrm{d} Y_{1}}{\mathrm{~d} z}=0 .
\end{gathered}
$$

Upstream. the eigenvalue problem has variable coefficients which would require a numerical solution. If $m_{o}$ upstream of the flame sheet is replaced by the value at the flame sheet, $m_{b}$, it can be readily solved. This simplification gives the same dispersion relation as Margolis (1980). As a result of the density-weighted axial coordinate, Margolis' result corresponds to ours when a value of $m,=m$, is used. When a densityweighted coordinate system is used, it is necessary to assume that $\rho^{2} D$ and $k p$ are constant in order to keep the coefficients constant; the present approach only requires that $\rho D$ is constant.

It can be shown that the eigenvalues of the variable coefficient eigenvalue problem are bounded from above and below by replacing the coefficient $m_{o}(z)$ by $m_{u}$ and m. Consider eq. (39). At the critical point, the real part of tu is zero. Two possibilities exist, the imaginary part could also be zero or the imaginary part could be non-zero. In the case of a principal exchange of stability, $w$ is identical zero and the problem of a variable coefficient no longer exists (for $z<f$. $m u$ is taken constant). In the case of a Hopf bifurcation, multiply eq. (39j with to* $Y_{1}^{*}$ (conjugates) and integrate between $z=-\mathrm{h}$ and $z=f^{-}$. If the integrals $I$, and $I$, are defined as follows:

$$
\begin{aligned}
& I_{1}=\int_{-h}^{F^{-}} \omega^{*} Y_{1}^{*}\left[\frac{1}{L e} \frac{\mathrm{d}^{2} Y_{1}}{\mathrm{~d} z^{2}}-x \frac{\mathrm{d} Y_{1}}{\mathrm{~d} z}\right] \mathrm{d} z \\
& I_{2}=\int_{-h}^{F^{-}} m_{o}\left|Y_{1}\right| \mathrm{d} z
\end{aligned}
$$

one can write

$$
|\omega|=\frac{\operatorname{Re}\left(I_{1}\right)}{I_{2}} .
$$

In $I_{2}$ the positive real function $\left|Y_{1}\right|$ is multiplied with the positive monotone decreasing function $m$. If $I$, and $I$, denote the integrals of $m_{u}\left|Y_{1}\right|$ and $m_{b}\left|Y_{1}\right|$ 
over the same interval. the following inequalities result:

$$
I_{3}>I_{2}>I_{4}
$$

and hence

$$
\left|\omega_{4}\right|>|\omega|>\left|\omega_{3}\right|
$$

The adiabatic value $m_{a}<\min \left(m_{0}\right)=\mathrm{m}$," and if $\mathrm{m}$, would be used, the absolute value of the eigenvalue for $-\mathrm{h} \leqslant z \geqslant 0$ will be overestimated.

The flame is located very close to the inlet of the burner. The wavelength of the pressure perturbation is large in comparison to the stand-off distance so that it is assumed that the pressure between the inlet of the burner and the flame sheet remains constant. If the pressure is constant upstream of the flame sheet, then $\mathrm{p} ; \quad=0$.

The upstream mass flux is also taken as constant and these assumptions decouple the temperature and species balances [eqs (39) and (40)] from the rest of the eigenvalue problem and allows the separate solution of $\mathrm{V}$ and $\mathrm{Y}$ both upstream and downstream. In particular? the solutions for $\theta_{1}$ and $Y_{1}$ are given by

$$
\begin{aligned}
& \theta_{1}^{-}=\mathrm{Ae}^{\lambda_{1} z+\omega \tau}+B \mathrm{e}^{\lambda_{2} z+\omega \tau} \\
& \theta_{1}^{+}=\mathrm{Ce}^{\lambda_{1} z+\omega \tau} \\
& Y_{1}^{-}=D \mathrm{e}^{\lambda_{1} z+\omega \tau}+G \mathrm{e}^{v_{2} z+\omega \tau} \\
& Y_{1}^{+}=0
\end{aligned}
$$

where

$$
\begin{aligned}
& \lambda_{1,2}=\frac{1}{2}\left(x_{F} \pm \sqrt{\left(x_{F}\right)^{2}+4 \omega m_{b}}\right) \\
& v_{1,2}=\frac{L e}{2}\left(x_{F} \pm \sqrt{\left(x_{F}\right)^{2}+\frac{4 \omega m_{b}}{L e}}\right)
\end{aligned}
$$

and $\lambda_{1}$ is the root with the negative real part. Making use of eqs (41)-(44), (47) and (48) and eliminating coefficients $A, \mathrm{~B}, \mathrm{C}, \mathrm{D}$ and $\mathrm{G}$ yields the following dispersion relation:
The particular solutions are given by

$$
\begin{aligned}
& m_{1 p}^{+}=H \mathrm{e}^{j_{2} z+\omega \tau} \\
& p_{1 p}^{+}=J \mathrm{e}^{j_{z} z+\omega \tau} \\
& u_{1 p}^{+}=K \mathrm{e}^{\dot{j}_{2} z+\omega \tau} .
\end{aligned}
$$

The functions $m_{1 p}^{+}, p_{i p}^{+}$and $u_{1 p}^{+}$can be written in terms of $\theta_{1}^{+}$. The homogeneous solutions for $u_{1}^{+}, m_{1}^{+}$and $p_{1}^{+}$are

$$
\begin{gathered}
u_{1 h}^{+}=M \mathrm{e}^{\lambda_{3} z+\omega \tau}+N \mathrm{e}^{\lambda_{4} z+\omega \tau} \\
m_{1 h}^{+}=-\frac{\rho_{0}^{+} \lambda_{3}}{\omega+u_{0} i_{3}} M \mathrm{e}^{\lambda_{3} z+\omega t}-\frac{\rho_{o}^{+} \lambda_{+}}{\omega+u_{o} i_{4}} N \mathrm{e}^{\lambda_{4} z+\omega \tau}
\end{gathered}
$$

$p_{1 h}^{+}=-\frac{p_{0}^{+} \lambda_{3}}{\omega+u_{0} i_{3}} M \mathrm{e}^{\lambda_{3} z+\omega \tau}-\frac{p_{0}^{+} \lambda_{4}}{\omega+u_{0} i_{4}} N \mathrm{e}^{i_{4} z+\omega r \tau}$

where

$$
i_{3,+}=\frac{-2 x_{F} \omega \pm \sqrt{\left(2 \gamma_{F}(\omega)^{2}-4\left(x_{F} u_{o}^{+}-x_{1}\right)\left(\rho_{o}^{+} \omega^{2}\right)\right.}}{2\left(x_{F} u_{o}-x_{1}\right)}
$$

Note: An eigenvalue problem can only be solved within an arbitrary constant. In this study all variables are scaled with respect to $f_{11}$.

\subsection{Coupled problem}

The coupled linear stability analysis problem consists of the uncoupled equations (36)-(40) with the energy balance replaced by

$m_{o} \omega \theta_{1}+x \frac{\mathrm{d} \theta_{1}}{\mathrm{~d} z}=\frac{\mathrm{d}^{2} \theta_{1}}{\mathrm{~d} z^{2}}+\frac{R}{C_{p}}\left(m_{o} \frac{\mathrm{d} \theta_{1}}{\mathrm{~d} \tau}+\theta_{o} \frac{\mathrm{d} m_{1}}{\mathrm{~d} \tau}\right)$.

The continuity and jump conditions and the boundary conditions of the coupled problem [eqs (41)-(48)] hold, with an additional boundary condition at the

$$
\begin{aligned}
& \frac{-v_{1}\left[x_{F} L e-v_{2}\right] \mathrm{e}^{-v_{2} h}+v_{2}\left[x_{F} L e-v_{1}\right] \mathrm{e}^{-v_{1} h}}{\left(x_{F} L e-v_{2}\right) \mathrm{e}^{-i_{2} h}-\left(x_{F} L e-v_{1}\right) \mathrm{e}^{-v_{1} h}}+x_{F} L e \\
& =\frac{\gamma}{2 \theta_{b}^{2}}\left[\frac{\mathrm{e}^{-i_{1} h}-\mathrm{e}^{-\lambda_{2} h} x_{F}\left(1-\theta_{u}\right)\left(x_{F}-i_{2}-x_{F} \gamma\left(1-\theta_{u}\right) / 2 \theta_{b}^{2}\right)}{\left(i_{1}-i_{2}-x_{F} \gamma\left(1-\theta_{u}\right) / 2 \theta_{b}^{2}\right) \mathrm{e}^{-i_{2} h}+\left(x_{F} \gamma\left(1-\theta_{u}\right) / 2 \theta_{b}^{2}\right) \mathrm{e}^{-i_{1} h}}+x_{F}\left(1-\theta_{u}\right)\right]
\end{aligned}
$$

The solutions for $m$; and 11 ; are found by means of eqs (36) and (37) as

$$
\begin{aligned}
& m_{1}^{-}=-\frac{m_{o}^{-}}{\theta_{o}^{-}}\left(A \mathrm{e}^{\dot{j}_{1} z+\omega \tau}+B \mathrm{e}^{\dot{j}_{2} z+\omega \tau}\right) \\
& u_{1}^{-}=\frac{u_{o}^{-}}{\theta_{o}^{-}}\left(A \mathrm{e}^{\dot{\lambda}_{1} z+\omega \tau}+B \mathrm{e}^{\dot{j}_{2} z+\omega \tau}\right)
\end{aligned}
$$

The downstream pressure perturbation. $\mathrm{p}$; is not identically zero. Since the downstream temperature perturbation is solved first, it becomes a non-homogeneous function in the eigenvalue problem (36)-(38). burner outlet, $z=\mathrm{L}-h$ :

$$
\frac{\mathrm{d} \theta_{1}}{\mathrm{~d} z}=0
$$

Note that the solutions to the coupled problem are distinguished from the uncoupled problem by the superscript ${ }^{*}$. The solutions for $\theta_{1}^{*}$ and $Y_{1}^{*}$ are given by

$$
\begin{gathered}
\theta_{1}^{*-}=A^{*} \mathrm{e}^{\lambda_{1}=+\omega \tau}+B^{*} \mathrm{e}^{\lambda_{2} z+\omega \tau} \\
\theta_{1}^{*+}=C^{*} \mathrm{e}^{\delta_{1} z+\omega \tau}+C_{2}^{*} \mathrm{e}^{\delta_{2} z+\omega \tau}+C_{3}^{*} \mathrm{e}^{\delta_{3} z+\omega \tau}
\end{gathered}
$$$$
\text { (aig } n+C_{+}^{*} \mathrm{e}^{\delta_{\alpha} z+\omega r}
$$ 


$$
\begin{gathered}
Y_{1}^{*-}=D^{*} \mathrm{e}^{v_{1} z+\omega z}+G^{*} \mathrm{e}^{v_{2} z+\omega r} \\
Y_{1}^{*+}=0
\end{gathered}
$$

where $\lambda_{1.2}$ are given by eq. (57), $v_{1.2}$ are given by eq. (58)and $\delta_{1,2.3,}$, are found by solving the following fourth-order polynomial:

$$
\begin{aligned}
& x_{1} \delta^{+}-\left[2 \theta_{o} u_{o}+x_{1} x_{F}+\frac{R}{C_{p}} u_{o}^{3}\right] \delta^{3} \\
& +\left[2 x_{F} \theta_{o} u_{o}-\left(x_{1} m_{v}+\frac{3 R}{C_{p}} u_{o}^{2}\right) \omega-\theta_{o} \omega^{2}\right] \delta^{2} \\
& \quad+\left[(\omega+2) x_{F} \theta_{o} \omega-\frac{3 R}{C_{p}} u_{o} \omega^{2}\right] \delta \\
& \quad+\left(m_{o} \theta_{o}+1\right) \omega^{3}=0 .
\end{aligned}
$$

The solutions for the upstream density and velocity perturbations are given by

$$
\begin{gathered}
m_{1}^{*-}=-\frac{m_{o}^{-}}{\theta_{o}^{-}}\left(A^{*} \mathrm{e}^{\lambda_{1} z+\omega \tau}+B^{*} \mathrm{e}^{\lambda_{2} z+\omega \tau}\right) \\
u_{1}^{*-}=\frac{u_{o}^{-}}{\theta_{o}^{-}}\left(A^{*} \mathrm{e}^{\lambda_{1} z+\omega \tau}+B^{*} \mathrm{e}^{\lambda_{2} z+\omega \tau}\right) .
\end{gathered}
$$

Using eqs (36)-(38) we can write

$$
\begin{aligned}
& m_{1}^{*+}=\left(\frac{m_{o} x_{1} \delta^{2}}{\left(\omega+u_{o} \delta\right)^{2}-\theta_{o} x_{1} \delta^{2}}\right) \theta_{1}^{*+} \\
& m_{1}^{*+}=\left(\frac{m_{o} x_{1} \delta_{1}^{2}}{\left(\omega+u_{o} \delta_{1}\right)^{2}-\theta_{o} \alpha_{1} \delta_{1}^{2}}\right) C_{1}^{*} \mathrm{e}^{\delta_{1} z+\omega r}
\end{aligned}
$$

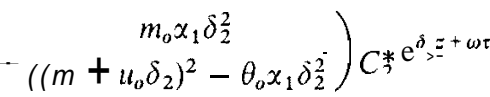

$$
\begin{aligned}
& +\left(\frac{m_{o} x_{1} \delta_{3}^{2}}{\left(\omega+u_{o} \delta_{3}\right)^{2}-\theta_{o} x_{3} \delta_{1}^{2}}\right) C_{3}^{*} \mathrm{e}^{\delta_{3} z+\omega r} \\
& \left.+\begin{array}{c}
m_{o} \alpha_{1} \delta_{4}^{2} \\
\left(\left(m+u_{0} \delta_{4}\right)^{2}-\theta_{0} \alpha_{1} \delta_{4}^{2}\right.
\end{array}\right) C_{4}^{*} \mathrm{e}^{\delta_{4} z+\omega r}
\end{aligned}
$$

and

$$
\begin{aligned}
u_{1}^{*+}= & \left(\frac{x_{1} \delta\left(\omega+u_{o} \delta\right)}{\theta_{o} \alpha_{1} \delta^{2}-\left(\omega+u_{o} \lambda^{2}\right.}\right) \theta_{1}^{*+} \\
u_{1}^{*+}= & \left(\frac{x_{1} \delta_{1}\left(\omega+u_{o} \delta_{1}\right)}{\theta_{o} x_{1} \delta_{1}^{2}-\left(\omega+u_{o} j^{2}\right.}\right) C_{1}^{*} \mathrm{e}^{\delta_{1}=+\omega \tau} \\
& +\left(\frac{x_{1} \delta_{2}\left(\omega+u_{o} \delta_{2}\right)}{\theta_{o} x_{1} \delta_{2}^{2}-\left(\omega+u_{o}\right)^{2}}\right) C_{2}^{*} \mathrm{e}^{\delta_{2}=+\omega \tau} \\
& +\left(\frac{x_{1} \delta_{3}\left(\omega+u_{o} \delta_{3}\right)}{\theta_{o} x_{1} \delta_{3}^{2}-\left(\omega+u_{o}\right)^{2}}\right) C_{3}^{*} \mathrm{e}^{\delta_{3} z+\omega \tau} \\
& +\left(\frac{x_{1} \delta_{4}\left(\omega+u_{o} \delta_{4}\right)}{\theta_{o} \chi_{1} \delta_{4}^{2}-\left(\omega+u_{o} j\right)^{2}}\right) C_{4}^{*} \mathrm{e}^{\delta_{+}=+\omega \tau} .
\end{aligned}
$$

Using eqs (41)-(48) and (70) and eliminating coefficients $A^{*}, \mathrm{~B}^{*}, C_{1}^{*}, C_{2}^{*}, C_{3}^{*}, C_{4}^{*}, D^{*}$ and $G^{*}$ yields the coupled problem dispersion relation. The dispersion relation is very lengthy and is not given here.

\section{RESULTS AND DISCUSSION}

The basic solution is stable for flame temperatures between the adiabatic temperature $T_{a}$ (upper open boundary) and the critical bifurcation temperature $T_{\mathrm{c}}$ (lower bound). The dimensionless critical bifurcation temperature is denoted as $\theta_{\mathrm{c}}$.

In Fig. 2. B, is plotted as a function of the inlet burner temperature for $L e=1.5$ and $L e=2.5$. The curves are for an inlet fuel fraction of $6 \%$. For the case where $L e=2.5$ results are shown for both the coupled and uncoupled model. It is evident that the uncoupled model exhibits much the same behaviour as the coupled model. The uncoupled model results in slightly lower critical temperatures. No solution was found for $L e=1.5$ with the uncoupled model. For the coupled model however, a solution was found for $L e=1.5$, which indicates that the flame can become unstable at this lower Lewis number. It is important to note that the uncoupled model does not predict instability for Lewis numbers lower than 2, but with the uncoupled model we see a definite range of inlet burner temperatures where the flame becomes unstable at a Lewis number of 1.5 . The range of inlet burner temperatures is smaller than in the uncoupled case, and ranges from $\mathrm{T},=400$ to $460 \mathrm{~K}$. Also evident is that lower burner temperatures have higher critical temperatures. Since the adiabatic temperature depends on the burner temperature, the stable region is decreased when the burner temperature is lowered.

Figure 3 illustrates the effect of inlet fuel fraction on the critical temperature. The burner temperature is $470 \mathrm{~K}$ and the uncoupled model was used. The lower Lewis number has a larger region of stability, i.e. the region between the adiabatic temperature $\left(\theta_{a}=1\right)$ and the critical bifurcation temperature $B$, The region of stability is increased with an increase in fuel fraction. It is stable over a smaller range of inlet fuel fractions than for $L e=2.5$.

The frequency of the oscillation at the Hopf bifurcation point is shown in Figs 4(a) and (b) for both the coupled and uncoupled models, and a Lewis number of 2.5. It is presented in $\mathrm{Hz}$ and note that the values lie in the audible range. At low inlet fuel concentrations

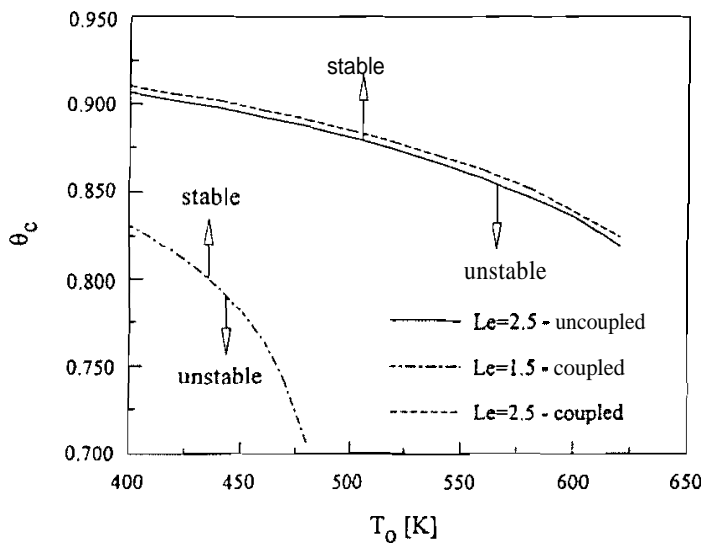

Fig. 2. Critical temperature (6\% methane) 
the frequencies are low as well, but it increases as the fuel concentration is increased from 3 to $8 \%$. This sensitivity is ideal for control applications. The frequencies are much higher for the lower burner temperature $\left(T_{o}=470 \mathrm{~K}\right)$.

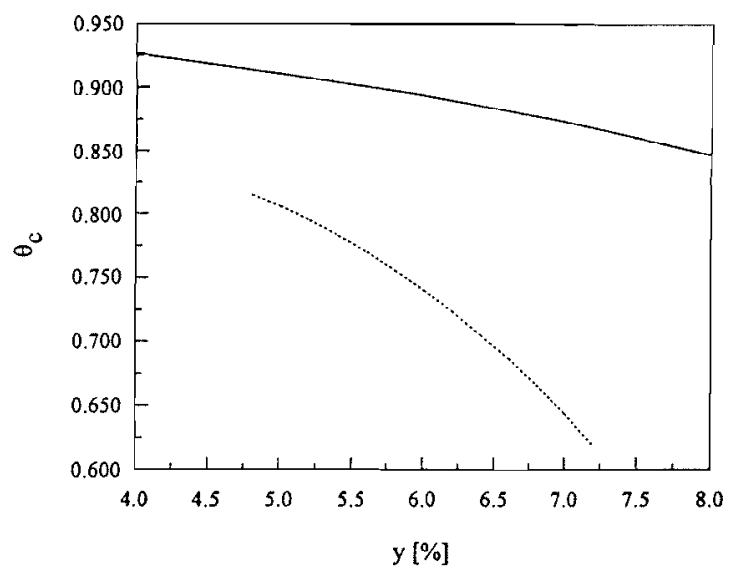

Fig. 3. Critical temperature $T_{v}=470 \mathrm{~K}$.

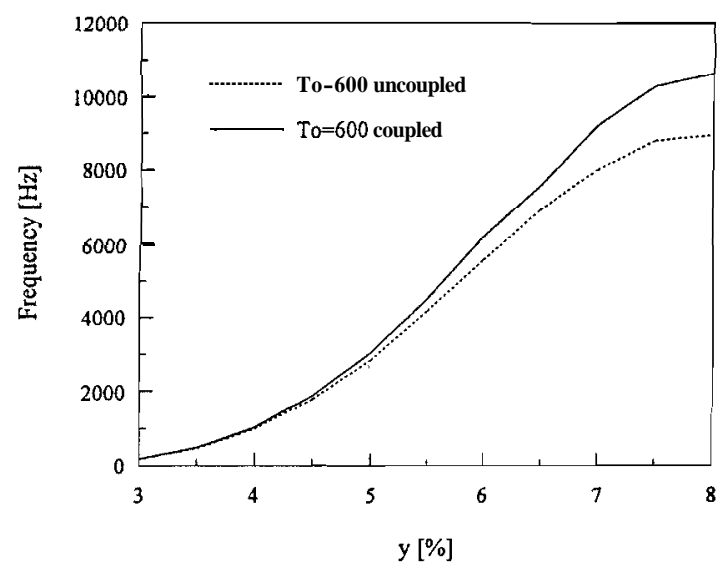

Fig. 4(a). Frequency of flame oscillation $T_{,}=600 \mathrm{~K}$.

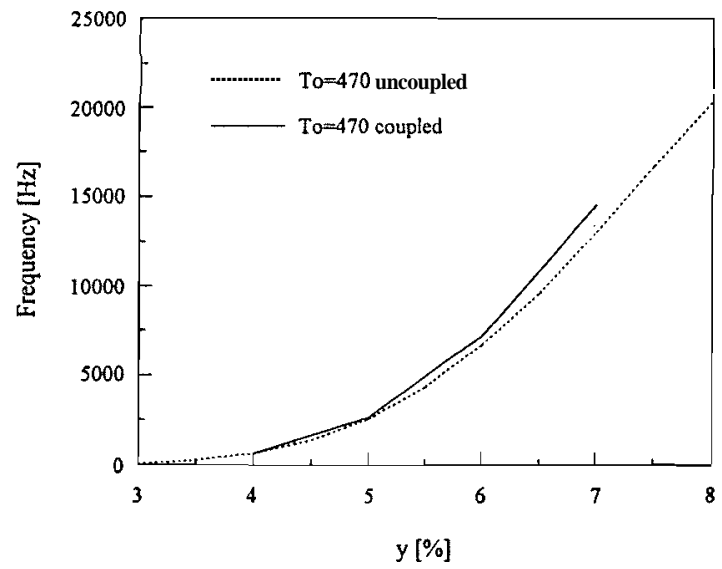

Fig. 4(b). Frequency of flame oscillation $T_{v}=600 \mathrm{~K}$.
In Fig. 5 the frequencies are shown for the coupled model and two burner temperatures (Lewis number of 1.5). The frequencies are much lower for this Lewis number. Higher burner temperature results in much lower frequencies - for a fuel fraction of $6 \%$ increasing the burner temperature from 450 to $470 \mathrm{~K}$ decreases the frequency from 210 to $75 \mathrm{~Hz}$.

In Figs 6 and 7 the pressure perturbation is shown in the vicinity of the flame sheet and far downstream, respectively, for the uncoupled model. The particular part of the solution decays very rapidly downstream. In Fig. 6 the variation in the flame position is shown over one period. Close to the flame the particular solution determines the pressure variation but it approaches the value of the homogeneous solution after a few length scale units. The spatial behaviour of the homogeneous solution is nearly periodical, but the wavelength is quite large on the dimensionless length scale. Therefore, a dimensional scale is used in Fig. 7 to show the behaviour far downstream for the dimensionless times shown. A standing wave pattern develops with fixed nodes. However, there is modulation of this wave pattern. Due to the large value of

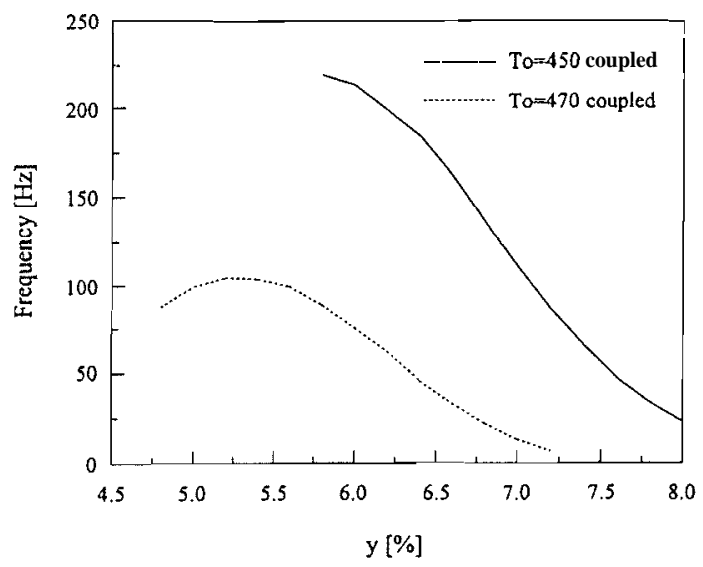

Fig. 5. Frequency of flame oscillation $\mathrm{Le}=1.5$

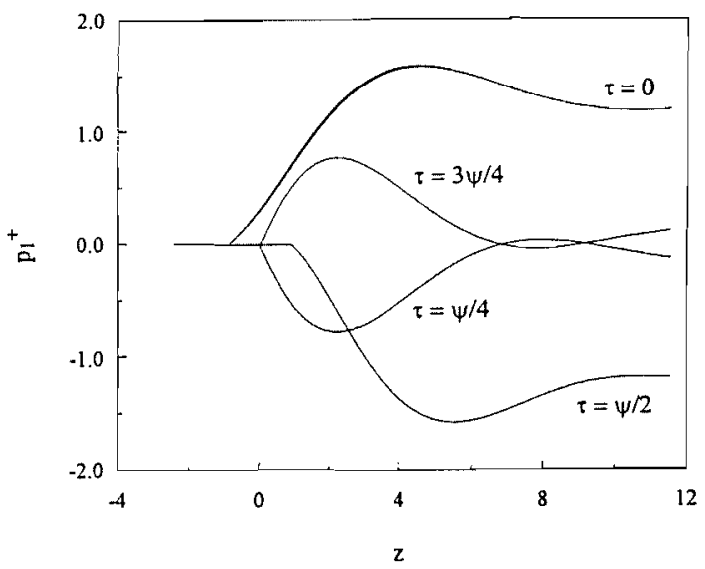

Fig. 6. Pressure perturbation (p;) far downstream 


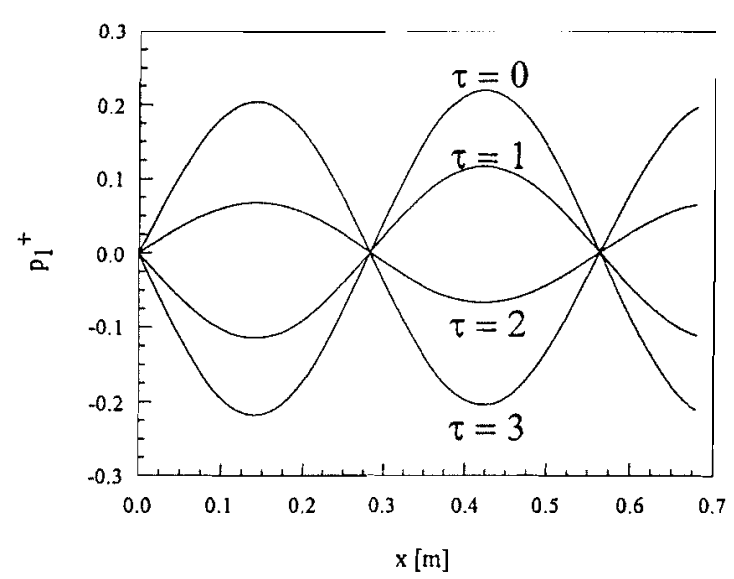

Fig. 7. Pressure perturbation (p;) for smaller $x_{1}$

the acoustic perturbations can be driven by a flame instability, without any external forcing involved. The frequencies of the perturbations have been reported for typical parameter values. The frequencies vary over a large range when the inlet fuel concentration is varied. When viscosity is considered in the analysis, the pressure perturbation can be amplified. Increased adiabatic flame velocities lead to smaller $x_{1}$ values and stronger modulation and amplification.

\section{Acknowledgement}

The authors gratefully acknowledge the financial support of the National Science Foundation through grant CTS9308813 and OSR-95-952250 as well as the Center for Materials Research and Analysis at the University of NebraskaLincoln.

\section{NOTATION}

C concentration

$x_{1}$ for typical subsonic flames, it follows from eq. (68) C, heatcapacity that the roots $\lambda_{3,4}$ are pure imaginary and they are not complex conjugates. When $x_{1}$ is decreased, the difference between the two roots become bigger and the modulation becomes more pronounced.

If the viscosity term is included in the momentum balance, eq. (38) becomes

$$
\begin{aligned}
m_{o}\left[\omega u_{1}+u_{o} \frac{\mathrm{d} u_{1}}{\mathrm{~d} z}\right] & +\left(m_{1} u_{o}+m_{o} u_{1}\right) \frac{\mathrm{d} u_{o}}{\mathrm{~d} z} \\
& +x_{1} \frac{\mathrm{d} p_{1}}{\mathrm{~d} z}=\operatorname{Pr} \frac{\mathrm{d}^{2} u_{1}}{\mathrm{~d} z}
\end{aligned}
$$

where $\boldsymbol{P r}$ denotes the Prandtl number $\mu C_{p} / k_{c}$. As was shown before, the particular solutions are only significant close to the flame sheet. However, the homogeneous parts of $u_{1}^{+}, m_{1}^{+}$and $p_{1}^{+}$are affected by the viscosity term. The characteristic equation, whose roots are shown in eq. (68), now becomes a cubic polynomial with complex coefficients;

$$
\begin{aligned}
\left(-\operatorname{Pr} u_{o}^{+}\right) \lambda^{3} & +\left(x_{F} u_{o}^{+}-\operatorname{Pr} w-x_{1}\right) \lambda^{2} \\
& +2 \omega x_{F} \lambda+\omega^{2} m_{o}^{+}=0 .
\end{aligned}
$$

The third root has a large negative real part, but the other two roots are very close to the roots of eq. (68) The important role of the $\boldsymbol{P r}$ number is to change the roots from pure imaginary values to complex roots with non-zero real parts. Although one of the roots has a very small positive real part, this term now has the ability to amplify as $z$ increases. It must be kept in mind that a length scale based on the thermal length has been chosen, this length has typical values of $10-' \mathrm{~m}$. If downstream distances of $50-100 \mathrm{~cm}$ are considered, the dimensionless values become large and the products of these values and the small positive real part could become significant.

This analysis lays the basis for a control strategy of flames, based on acoustic perturbations which develop downstream of the flame. It has been shown that

D

$\begin{array}{ll}D a & \left(=\frac{\kappa k e^{-\gamma / \theta_{b}}}{U_{u}^{2}}\right) \\ E & \text { activation energy } \\ f & \text { instantaneous flame sheet position } \\ \Delta H & \text { heat of reaction } \\ h & \text { distance between burner and flame sheet } \\ k & \text { frequency factor } \\ k_{c} & \text { thermal conductivity } \\ L & \text { tube length } \\ L e & (=\kappa / D)\end{array}$

$m \quad$ dimensionless density

$M_{\text {air }} \quad$ molar mass of air $p$ $P$ $R_{g} \quad$ molar gass constant

$R_{m} \quad$ mass gass constant

$t$ time

$T$ temperature

$T_{c} \quad$ critical bifurcation temperature

$u$ dimensionless gas velocity

$U \quad$ gas velocity

$U_{u} \quad$ adiabatic flame velocity

$x \quad$ axial variable

$y \quad$ molar fuel fraction

$Y \quad$ dimensionless mass fuel fraction

$z \quad$ dimensionless axial variable

\section{Greek letters}

$\begin{array}{ll}x_{F} & \text { dimensionless flux } m_{o} t_{o} \\ x_{1} & \left(=R_{m} T_{u} / U_{a}^{2}\right) \\ \gamma & \left(=E / R_{m} T_{a}\right) \\ \delta_{F} & \text { Kronecker delta function } \\ \theta & \text { dimensionless temperature } \\ \theta_{b} & \text { dimensionless temperature at flame sheet } \\ \theta_{u} & \text { dimensionless temperature at burner } \\ K & \left(=k_{c} / \rho_{a} C_{p}\right) \\ P & \text { density } \\ \tau & \text { dimensionless time }\end{array}$




\section{REFERENCES}

Afanas'ev, V. V., Abrukov, S. A., Kidin, N. I. and Kuz'min, A. K. (1995).Conditions for theexcitation of a laminar kinetic singing flame. Combustion Explosion Shock Waves 31(4), 432-436.

Clavin, P., Pelce, P. and Longtin, H. (1990) Onedimensional vibratory instability of planar flames propagating in tubes. J. Fluid Mech. 216, 299-322.

Garris, C. A., Toong, T. Y. and Patureau, J. P. (1975) Chemi-acoustic instability structure in irreversibly reacting systems. Acta Astronaut. 2, 981-997.

Gilbert, R. G., Ortoleva, P. J. and Ross, J. (1973) Nonequilibrium relaxation methods. Acoustic effects in transient chemical reactions. J. Chetn. Phys. $58,3625-3633$.

Joulin, G. and Clavin, P. (1979)Linear stability analysis of nonadiabatic flames: diffusional-thermal model. Combrrst. Flame 35, 139-153.

Lawn, C. J. (1982)Criteria for acoustic pressure oscillations to be driven by a diffusion flame. Proceedings of the 19th Symposium (International) on Combustion, The Combustion Institute, pp. 237-244.

McIntosh, A. C. (1987) Combustion-acoustic interaction of a flat flame burner system enclosed within an open tube. Combust. Sci. Technol. 54, $217-236$.
Macquisten, M. A. and Dowling. A. P. (1995) Combustion oscillations in a twin-stream afterburner. $\mathbf{J}$. Sound Vibr. 188(4), 545-560.

Margolis, S. B. (1980) Bifurcation phenomena in burner-stabilized premixed flames. Combust. Sci. Technol. 22, 143-169.

Margolis, S. B. (1993) Nonlinear stability of combustion-driven acoustic oscillations in resonance tubes. J. Fluid Mech. 253, 67-103.

Sipowicz, W. W., Ryan, N. W. and Baer. A. D. (1971) Combustion-driven acoustic oscillations in a gasfired burner. Proceedings of the 13th Sympositum (International) on Combustion, The Combustion Institute, pp. 559-564.

Sivashinsky, G. I. (1983) Instabilities, pattern formation, and turbulence in flames. Ann. Rev. Fluid Mech. 15, 179-199.

Sugimoto, T. and Matsui, Y. (1982)An experimental study on the dynamic behavior of premixed laminar flames. Proceeding of the 19th Symposium (International) on Cornbrrstion, The Combustion Institute, pp. 245-250.

Toong, T. Y., Arbeau, P., Garris, C. A. and Patureau, J. P. (1975) Acoustic-kinetic interactions in an irreversably reacting medium. Proceedings of the 15 th Symposium (International) on Combustion, pp. 87-100.

Williams, F. A. (1985)Combustion Theory (2nd ed.). Addison-Wesley, Reading, MA. 